\title{
Electrochemical Sensors and Biosensors
}

\author{
Farnoush Faridbod, ${ }^{1}$ Vinod Kumar Gupta, ${ }^{2,3}$ and Hassan Ali Zamani ${ }^{4}$ \\ ${ }^{1}$ Endocrinology and Metabolism Research Center, Tehran University of Medical Sciences, Tehran 1411413137, Iran \\ ${ }^{2}$ Department of Chemistry, Indian Institute of Technology Roorkee, Roorkee 247 667, India \\ ${ }^{3}$ Chemistry Department, King Fahd University of Petroleum and Minerals, Dhahran 31261, Saudi Arabia \\ ${ }^{4}$ Department of Applied Chemistry, Mashhad Branch, Islamic Azad University, Mashhad, Iran
}

Correspondence should be addressed to Farnoush Faridbod, faridbodf@tums.ac.ir

Received 19 December 2011; Accepted 20 December 2011

Copyright (C) 2011 Farnoush Faridbod et al. This is an open access article distributed under the Creative Commons Attribution License, which permits unrestricted use, distribution, and reproduction in any medium, provided the original work is properly cited.

Electrochemical sensors and biosensors have recently found extensive applications in diverse industries. Nowadays, many analytical instruments used in environmental, food, pharmaceutical, or clinical laboratories and also most of the commercial point-of-care devices work using chemical sensors or biosensors, as a whole or a basic part. Glucose biosensors used widely in glucometers and $\mathrm{pH}$ electrodes are the important and known examples of the electrochemical sensors. Day by day, the numbers of sensors or biosensors coming from the bench of research laboratories to the shelf of the commercial markets are increasing. Due to the high demand of the world market and human interest for having a device to check the concentration of species in different samples, simple and fast, in recent years, a hard competition on design and construct of new sensors and biosensors has occurred among the researchers.

Because of such an importance and to show various applications of this kind of devices, the topic of this special issue was devoted to electrochemical sensors and biosensors. Electrochemical sensors and biosensors can offer advantages of low detection limits, a wide linear response range, and good stability and reproducibility.

An electrochemical sensor is a device that transforms electrochemical information into an analytically useful signal. Electrochemical sensors usually composed of two basic components, a chemical (molecular) recognition system which is the most important part of a sensor and a physicochemical transducer which is a device that converts the chemical response into a signal that can be detected by modern electrical instrumentations. These two parts form a working (or sensing) electrode. A reference electrode and sometimes a counter electrode are also used in electrical measurements. Biosensors are chemical sensors in which the recognition system utilizes a biochemical mechanism.

Transduction of a biological or chemical signal into an electrical signal can be done by amperometry, voltammetry, potentiometry, or conductometry.

Next generation of sensor or biosensors will require considerable improvements in sensitivity, selectivity, and accuracy to meet the future needs in diversity of fields. Today, application of different nanoparticles in construction of sensors and biosensors as a modifier causes to approach to this purpose. The nanoparticles have different effects on response of the sensor or biosensor besides improving their thermal, electrical, and mechanical properties.

The papers selected for this special issue represent different kind of electrochemical sensing, different sensing materials, and also various nanoparticles used in determination various species. Although the papers are not an exhaustive representation of all area of electrochemical sensing or biosensing, the papers can give the readers an idea how to make a sensor or biosensor for different applications using electrochemical methods.

This special issue contains 15 papers, where 8 papers reported new design biosensors for different biological molecules. In one of them recent advances in electrochemical aptamer-based biosensors have been discussed.

Most of the papers have used nanoparticles in construction of their sensors or biosensors.

Three of sensors have applied voltammetric method for transduction of the sensor signal, and three ones have reported new designed potentiometric sensors.

One paper has used a chemically modified electrochemiluminescence sensor for determination of atropine sulfate in capillary electrophoresis system. 
At last, we would like to thank the authors for their admirable contributions and patience in publishing this special issue. Also, the fundamental works of all reviewers on these papers are sincerely acknowledged.

Farnoush Faridbod Vinod Kumar Gupta

Hassan Ali Zamani 


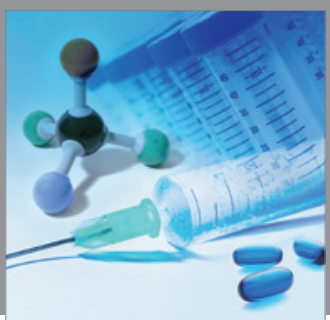

International Journal of

Medicinal Chemistry

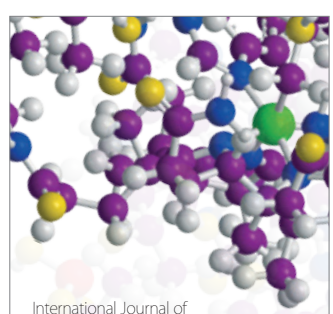

Carbohydrate Chemistry

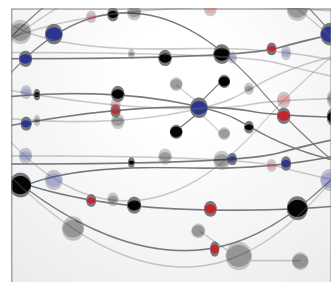

The Scientific World Journal
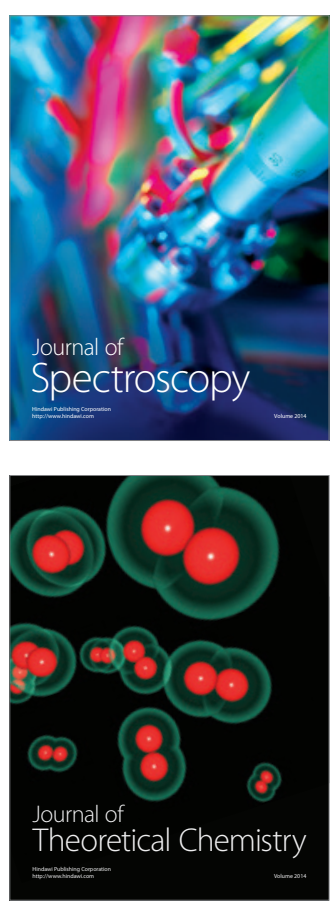
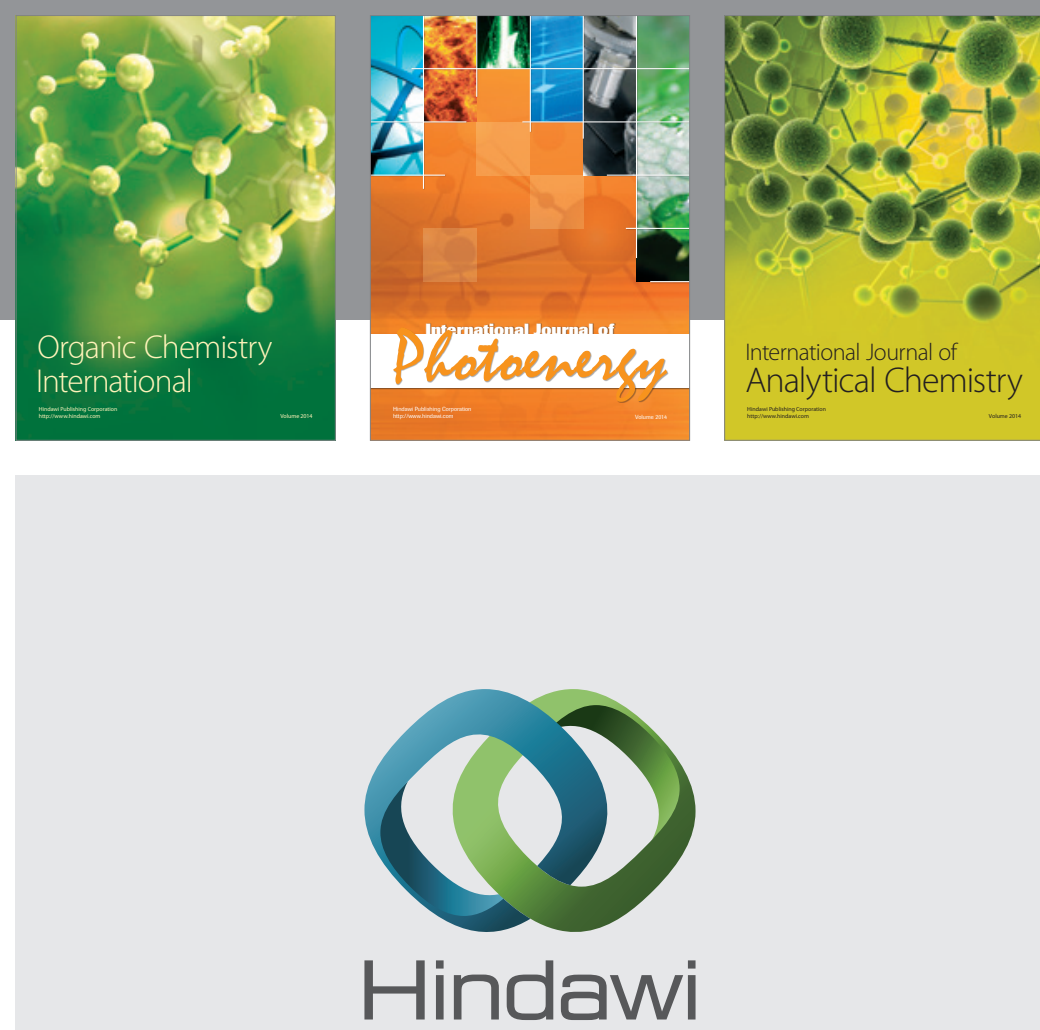

Submit your manuscripts at

http://www.hindawi.com
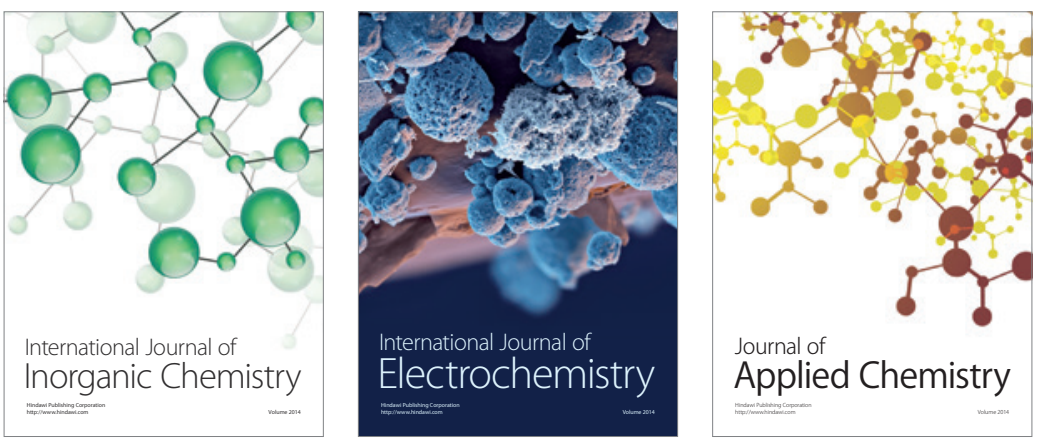

Journal of

Applied Chemistry
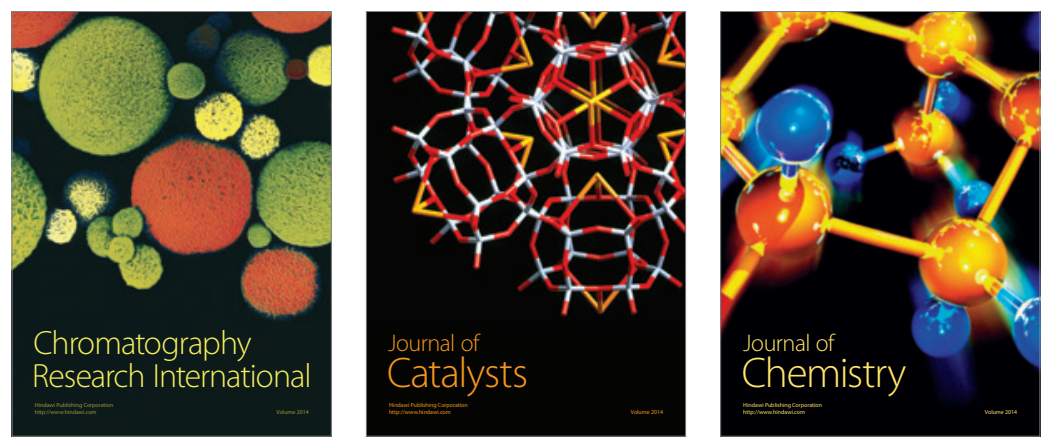
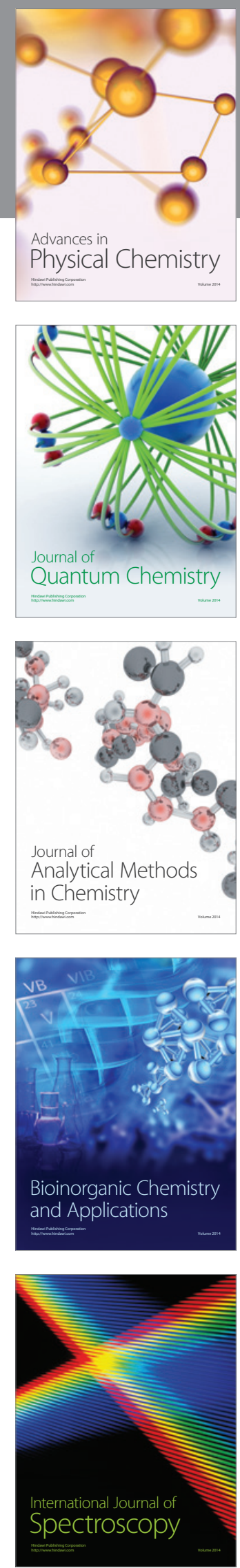